\title{
THE INFLUENCE OF PROPAGATION THROUGH THE IRREGULAR INTERSTELIAR PLASMA ON VLBI OBSERVATIONS .
}

\author{
B.J.Rickett and W.A.Coles, \\ Electrical and Computer Engineering Department, \\ University of California San Diego, \\ La Jolla, Ca 92093.
}

It has been known since pulsars were discovered that the interstellar medium (ISM) can cause angular broadening of radio sources. However, the amount of scattering along typical lines of sight to extra-galactic sources is not large enough to cause any loss of visibility, even on intercontinental baselines, unless the frequency is below about $100 \mathrm{MHz}$. Recent studies have shown that interstellar scattering (ISS) can have considerably stronger effects; this paper addresses their relevance to VLBI.

It is now known that there are two scales of fluctuation in the amplitude variations caused by ISS; a (smaller) diffractive scale and a (larger) refractive scale. For pulsars, which behave as effective point sources for ISS, both scales are observable, causing variability on times of the order of 10 minutes and 10 days, respectively. For extra-galactic sources, even the most compact have angular diameters larger by many orders of magnitude and only the longer term refractive fluctuations are detectable. Indeed such fluctuations are probably the major contribution to "low frequency variability", which had previously been ascribed to intrinsic variations (Rickett, Coles and Bourgois, 1984). Pulsar studies have been used to estimate the wavenumber spectrum of electron density in the ISM at scales of $10^{8}$ to $10^{11} \mathrm{~m}$ as a function of galactic coordinates (Rickett, 1977; Cordes, Weisberg and Boriakoff, 1985 - CWB; Hewish, Graham and Wolszczan, 1985; Blandford and Narayan, 1985; Cordes and Wolszczan, 1986; Cordes, Lovelace and Pidwerbetsky, 1986; Coles et a.l. 1987). Three aspects are relevant to VLBI observations.

First, there are lines of sight through the Galaxy that suffer greatly enhanced scattering (by many orders of magnitude), particularly at low galactic latitudes and through the inner galactic plane. Second, the unexpectedly strong refractive amplitude variations imply that there are random refractive position variations, which are possibly larger than the scattered image itself. Third, the form of the scattered image may, on occasion, take the form of a few discrete beams.

The enhanced scattering on particular lines of sight have already been 287

M. J. Reid and J. M. Moran (eds.), The Impact of VLBI on Astrophysics and Geophysics, 287-294.

(C) 1988 by the IAU. 
investigated in several VLBI onbservations, (e.g. Dennison et al. 1984). The physical site of the enhanced scattering is unknown. The evidence points to a low latitude distribution $( \pm 100 \mathrm{pc})$ of scattering "clouds", whose mean free path for intersection by a line of sight is about $1 \mathrm{kpc}$ (CWB). Specific structures may include the Cygnus loop (responsible for broadening CL4, Geldzahler and Shaffer, 1981). There is weak evidence for enhancements near other supernova remnants (Spangler et al. 1985). Also the source NGC 6334B is heavily scattered and lies in the same direction as a dense HII region and molecular cloud (Rodriguez et al. 1982). The enhanced scattering is to be compared with the background of a more uniform distribution modelled by a $\pm 500 \mathrm{pc}$ disc (CWB). The influence of scattering is to cause an (extra-galactic) point source to be scattered into an angular spectrum that has an instantaneous width $\theta_{\text {scat }}$ (FWHM) and to be displaced from the true source position by an angle $\theta_{\text {ref }} \propto \lambda^{2}$, which varies slowly and randomly with time. The scattering angle $\theta_{\text {scat }}$ is the most important parameter in determining the ISS effects on VLBI observations. The uniform scattering distribution corresponds to a typical scattering path $\mathrm{L}=0.5(\operatorname{cosec} \mathrm{b}) \mathrm{kpc}$ and a value:

$$
\theta_{\text {scat }} \sim 0.7\left(\mathrm{f}_{\mathrm{GHz}}\right)^{-2}(\operatorname{cosec} \mathrm{b})^{0.5} \text { mas. }
$$

This equation should be compared with the data in Figure 1 from Cordes, Ananthakrishnan and Dennison (1984), from which we have also estimated equation (1) above. In using it to estimate scattering on a given latitude and longitude one must expect very large upward deviations as the line of sight passes closer toward the inner galactic plane. Equation (1) should be a reasonable estimate down to latitudes of 20 degrees; at lower latitudes an increasing longitude dependence is expected and an increasing chance of encountering enhanced scattering, raising $\theta_{\text {scat }}$ by more than 2 orders of magnitude; see also Rao and Ananthakrishnan (1985).

We now discuss the conditions under which ISS will be resolvable in VLBI (i.e. when ISS will cause loss of visibility on a given baseline). We follow the approach of Cohen and Cronyn (1974) in discussing whether there will indeed be a loss of fringe visibility in a given situation. The basic condition, of course, is that the baseline should be longer than the field "coherence scale" so. Expressed in angular coordinates; the angular resolution of the instrument $\theta_{\text {res }}$ should be less than $\theta_{\text {scat }}=\left(k s_{0}\right)^{-1}$, where $k=2 \pi / \lambda$. with a long enough baseline, the amplitude and phase at the two antennas will be significantly different, differing by say 1 radian and $50 \%$ in amplitude over distance $s_{0}$. However for a point source, with no averaging due to bandwidth or integration time, the magnitude of the apparent visibility on a single baseline would be unity. There would also be a random phase. With a receiver bandwidth $B>$ $2 \mathrm{c} / \mathrm{L}\left(\theta_{\text {scat }}\right)^{2}$, or integration time $\mathrm{T}>\mathrm{s}_{\mathrm{O}} / \mathrm{V}$ or an intrinsic source diameter $\theta_{\text {source }}>s_{o} / L$, the visibility is reduced. Here $L$ is an effective path length through the scattering medium, estimated by $\mathrm{L}=$ $500 \mathrm{pc}(\operatorname{cosec} \mathrm{b})$ and $\mathrm{V}=50 \mathrm{~km} / \mathrm{s}$ is the velocity of the observer with respect to the diffraction pattern. The conditions are plotted in 
Figure 2, which displays the variables (a) $\theta_{\text {source }} f_{\mathrm{GHz}} \mathrm{L}_{\mathrm{kpc}}$; (b) $\mathrm{T}_{\min } \mathrm{f}_{\mathrm{GHz}}$; (c) $\mathrm{B}_{\mathrm{MHz}} \mathrm{L}_{\mathrm{kpc}}$ plotted against $\theta_{\text {scat. }}$. So for a given observation we need an estimate of the scattering angle, the path length and the instrumental quantities $\mathrm{f}_{\mathrm{GHz}}, \mathrm{B}_{\mathrm{MHz}}$ and $\mathrm{T}_{\mathrm{min}}$. There are separate vertical scales for each of the quantities (a), (b) and (c), which are each plotted as a point at the appropriate $\theta_{\text {scat }}$. The interferometer visibility will be reduced when any of the points lie above and to the right of the corresponding lines. It is clear from the figure that the most likely cause of the loss of visibility is angular diameter smearing of the diffraction pattern by different (incoherent) parts of the source, based on angular diameters greater than (say) 0.1 mas.

With a single baseline one can predict whether ISS can reduce the visibility as discussed above. There is also a random phase slope or position shift. However, this is usually given as a position difference from a calibration source. With many baselines a brightness distribution can be estimated. In general the source visibility over baseline $\gamma$ is multiplied by the visibility function due to scattering $\exp \left[-\left(\gamma / s_{0}\right)^{\beta-2}\right]$, where $\beta$ is the power law exponent defined below. Thus the source brightness distribution will be convolved with the "point spread function" due to scattering. With approprate averaging this is the Fourier transform of the visibility given above; it depends on the wavenumber spectrum of the ISM density irregularities $P_{N}(K)$, where $K$ is the wavenumber. A Kolmogorov spectrum has $\mathrm{P}_{\mathrm{N}}(\mathrm{K}) \propto\left(\mathrm{K}_{\mathrm{O}}^{2}+\mathrm{K}^{2}\right)-\beta / 2$ with $\beta=11 / 3$. In the absence of averaging, the detailed form of the image will show "speckles" down to the resolution limit. Any value of $\beta$ between 2 and 4 should give an essentially circular image of the form given above. If the spectrum were steeper ( $\beta$ between 4 and 6 ) several differences arise. First is the prominence of random refractive shifts by angles larger than $\theta_{\text {scat; }}$ thus the form of the instantaneous scattering spread function is different from the long term average that includes the image wander. It is thought that the form given above corresponds to the "instantaneous" image, though the question has not been resolved formally. Goodman and Narayan (1985) argue that the image will show a slowly changing ellipticity and may be broken down into a hierarchy of sub-images; their analysis, however, does not appear to address questions of averaging over the source or bandwidth, nor the form of the instantaneous image in the absence of refractive displacement.

On the observational side, there is only little evidence on the scattered image itself. The compact source at the Galactic Center has been resolved and has a wavelength dependent diameter, corresponding to very highly enhanced scattering. In addition the image is elongated by about 1.8:1 (Lo et al. 1985). There are severai other observations, which have shown a wavelength- squared dependence of diameter, but few have had a sufficient number of baselines to synthesize an image. See Spangler and Cordes, and Wilkinson, Spencer and Nelson (both in this volume) for recent measurements. Clues about the scattered image have also been assembled from observations of the dynamic ISS spectra of pulsars. The presence of multiple images has 
been inferred by Cordes and Wolszczan (1986). They suggest that there are times when the image consists of 2 or 3 discrete images, each broadened by scattering and separated from each other by several times their width. Such a situation is inconsistent with a simple Kolmogorov spectrum and requires either a steeper power spectrum (i.e. $\beta>4$ or a cut-off due to an inner scale) or the chance focussing action of discrete structures in the ISM, superimposed on a background of diffractive scattering. The latter explanation has recently been advanced (Romani, Blandford and Cordes, 1987) following the discovery of unexpected "focussing events" by Fiedler et al. (1987). It would be very interesting to detect such structures with VLBI techniques. In this context, the most convincing proof of a plasma scattering origin for a multiple image would be a dependence of their separation on the square of the wavelength.

We now need an advance in scattering theory to predict the statistical form of scattered images when many antennas are used and self-calibration or other techniques are used to eliminate antenna-based phase errors. The critical questions concern the rate at which scattering distortions of an image will change with time (as the line of sight traverses different sections of the ISM) or with differing angles across the source. We will discuss below apparent position wander, under the simplifying assumption that sufficient baselines have been used with self-cal to construct an image. We proceed by also assuming that the point source image does not change its shape with time and look for departures from the isoplanatic condition on a scale larger than that instantaneous image (i.e. distortions of the separation between components in the source). We examine only the contribution to this distortion from the irregular phase change obtained on a straight line integration through the medium - call this the geometric phase $\phi(x, y)$, where $(x, y)$ are coordinates transverse to the line of sight. The result will be reasonably accurate when the scattered image is well resolved; it is this case that we now examine.

Since a linear phase gradient is removed in self-cal (i.e. the image is centered) only position differences can be detected. Consider two point sources separated by $\Delta \theta$, we want to predict the magnitude of fluctuations in their apparent separation and how quickly such fluctuations change with time and with angle across the sky. This involves first and second order departures from a plane wavefront and depends on the form of the spectrum $\mathrm{P}_{N}(K)$. Using the representation of a thin screen at distance $L$, we examine the second difference of geometric phase:

$$
\begin{aligned}
& S(\gamma, \delta)=\left\langle[\phi(r)-\phi(x+\gamma)-\phi(x+\delta)+\phi(x+\delta+\gamma)]^{2}\right\rangle \text {, hence } \\
& \mathrm{S}(\gamma, \delta)=2 \mathrm{D}(\gamma)+2 \mathrm{D}(\delta)-\mathrm{D}(\delta+\gamma)-\mathrm{D}(\delta-\gamma) \text {, where } \\
& \mathrm{D}(\gamma)=\left\langle[\phi(\boldsymbol{x})-\phi(x+\gamma)]^{2}\right\rangle
\end{aligned}
$$

We consider the baseline magnitude $=\gamma>s_{0}$, so that the scattered image is resolved and $\delta=L \Delta \theta$. We find the error $\theta_{\text {ref }}$ in $\Delta \theta$ due to 
refractive distortion of the field using $k \theta_{\text {ref }} \sim$ (average difference over $\mathrm{L} \Delta \theta$ in the phase gradient over baseline $\gamma$ ), which gives $\theta_{\text {rgf }} \sim$ $[\mathrm{S}(\gamma, \mathrm{L} \Delta \theta)]^{0.5} / \mathrm{k} \gamma$. With the baseline limited to a maximum of $10^{\mathrm{r}} \mathrm{km}$ and $L \Delta \theta>L \theta_{\text {scat }}$, it follows that $\gamma \ll \delta$.

When the spectrum has a power law exponent $2<\beta<4, S(\gamma, \delta) \sim 2 \mathrm{D}(\gamma)$ independent of $\delta$. This gives $\theta_{\text {ref }} \sim \theta_{\text {res }}\left(\gamma / s_{0}\right)^{(\beta-2) / 2<} \theta_{\text {scat }}$. In words, the refractive error in the separation of sources $\Delta \theta$ is larger than the angular resolution $\theta_{\text {res }}$ of the interferometer and smaller than the scattering angle. We can also analyze the rate at which this changes either across the sky or with time as the line of sight intersects differing portions of the ISM, and find that the decorrelation angle for $\theta_{\text {ref }}$ is approximately equal to $\theta_{\text {ref }}$ itself, and the decorrelation time would be $L \theta_{\text {ref }} / \mathrm{V}$. With typical coherent integration times of minutes for VLBI, there would be detectable changes in component separation over times of the order months, but by angles smaller than the scatter broadening itself. As emphasized at the outset, in practice only the enhanced scattering is likely to be resolvable with earth-based interferometry and so too, refractive effects would only be important along such heavily scattered lines of sight.

For steeper power law exponents $4<\beta<6$, the quantity $S(\gamma, \delta)$ depends on both $\gamma$ and $\delta$, for $\gamma<\delta$; however, the phase gradient [S] ${ }^{0.5} / \gamma$ is then independent of $\gamma$, giving $\theta_{\text {ref }} \sim \theta_{\text {scat }}\left(\Delta \theta / \theta_{\text {scat }}\right)^{(\beta-4) / 2}$. Thus the refractive errors in separation are larger than the instantaneous scattering angle and increase with separation $\Delta \theta$, (slowly for $\beta \sim 4.3$ as has been proposed hy Goodman and Narayan, 1985). The angular decorrelation scale for the refractive errors would be $\Delta \theta$ and the decorrelation time would be $L \Delta \theta / \mathrm{V}$. The refractive errors would saturate at separations such that $L \Delta \theta \sim$ (outer scale of the ISM irregularities). The third possible wavenumber spectrum for the ISM that has been proposed involves an inner scale at about $10^{9} \mathrm{~m}$ (Coles et al. 1987). This scale is larger than $s_{o}$ and is specified in such a way that at the distance of a typical pulsar, the inner scale will cause interference - that is it will "focus". Under these conditions, that pertain to a frequency somewhat below $1 \mathrm{GHz}$, the scattering angle and refraction angle are about the same.

We conclude that there is indeed a predicted difference between the spectral models in regard to refractive distortion in the field of view; it is possible that low frequency VLBI may be able to resolve such differences and so provide a constraint on the wavenumber spectrum for the ISM. The measurements of Gwinn et al. in this volume have provided an upper limit on the amount of angular wander of 20 mas over 9 months in the Sag B2 $\mathrm{H}_{2} \mathrm{O}$ maser source, which has an apparently scatter-dominated diameter of about 0.3 mas. For the Kolmogorov type spectra we only predict that $\theta_{\text {ref }}<0.5$ mas. The $\beta=4.3$ model predicts $\theta_{\text {ref }} \sim \theta_{\text {scat }}\left(\mathrm{VT} / \mathrm{L} \theta_{\text {scat }}\right)(\beta-4) / \xi$. Taking an 8 kpc distance, the 9 -month observing time gives a refractive error about equal to the scattering angle. However, the critical calculation is how quickly does the refractive error change, since the true positions are not known. This 
will be not be dealt with here. In addition the issue of the shape of the scattered image of a point source may be measurable, though the intrinsic source averaging may smear such effects.

\section{REFERENCES}

Blandford, R.D. and Narayan, R.:1985, Mon. Not. Roy. Astro. Soc., 213, 591 .

Cohen, M.H. and Cronyn, W.M.:1974, Astrophys. J., 192, 193.

Coles, W.A., Frehlich, R.G., Rickett, B.J. and Codona, J.L.:1987, Astrophys. J., 315, 666 .

Cordes, J.M., Ananthakrishnan, S. and Dennison, B.:1984, Nature, 309, 689.

Cordes, J.M., Pidwerbetsky, and Lovelace, R.V.E.:1986, Astrophys. J., 310,737 .

Cordes, J.M. and Wolszczan, A.:1986, Astrophys. J. Lett., 307, L27.

Cordes, J.M., Weisberg, J.M. and Boriakoff, V.:1984, Astrophys. J., $288,221$.

Dennison, B., Thomas, M., Booth, R.S., Brown, R.L., Broderick, J.J and Condon, J.J.:1984, Astrophys. J., Astron. Astrophys., 135, 199.

Fiedler, R.L., Dennison, B., Johnston, K.J. and Hewish, A.:1987, Nature, in press.

Geldzahler, B.J. and Shaffer, D.B.:1981, Astrophys. J., 248, 132.

Goodman, J.J., and Narayan, R.:1985, Mon. Not. Roy. Astro. Soc., 214, 519 .

Hewish, A., Wolszczan, A. and Graham, D.:1985, Mon. Not. Roy. Astro. Soc., 213, 167 .

Lo, K.Y., Backer, D.C., Ekers, R.D., Kellerman, K.I., Reid, M.J. and Moran, J.M.:1985, Nature, 298, 825 .

Rao, A.P. and Ananthakrishnan, S.:1984, Nature, 312, 707.

Rickett, B.J.: 1977, Ann. Rev. Astron. Astrophys., 15, 479.

Rickett, B.J., Coles, W.A. and Bourgois, G.:1984, Astron. Astrophys., 134, 390 .

Rickett, B.J.: 1986, Astrophys. J., 307, 564.

Rodriguez, L.F., Canto, J. and Moran, J.M., Astrophys. J.:1982, 225, 103.

Romani, R.W., Blandford, R.D. and Cordes, J.M.:1987, submitted to Nature.

Spangler, S.R., Mutel, R.L., Benson, J.M. and Cordes, J.M.:1985, Astrophys. J., 301, 312 . 


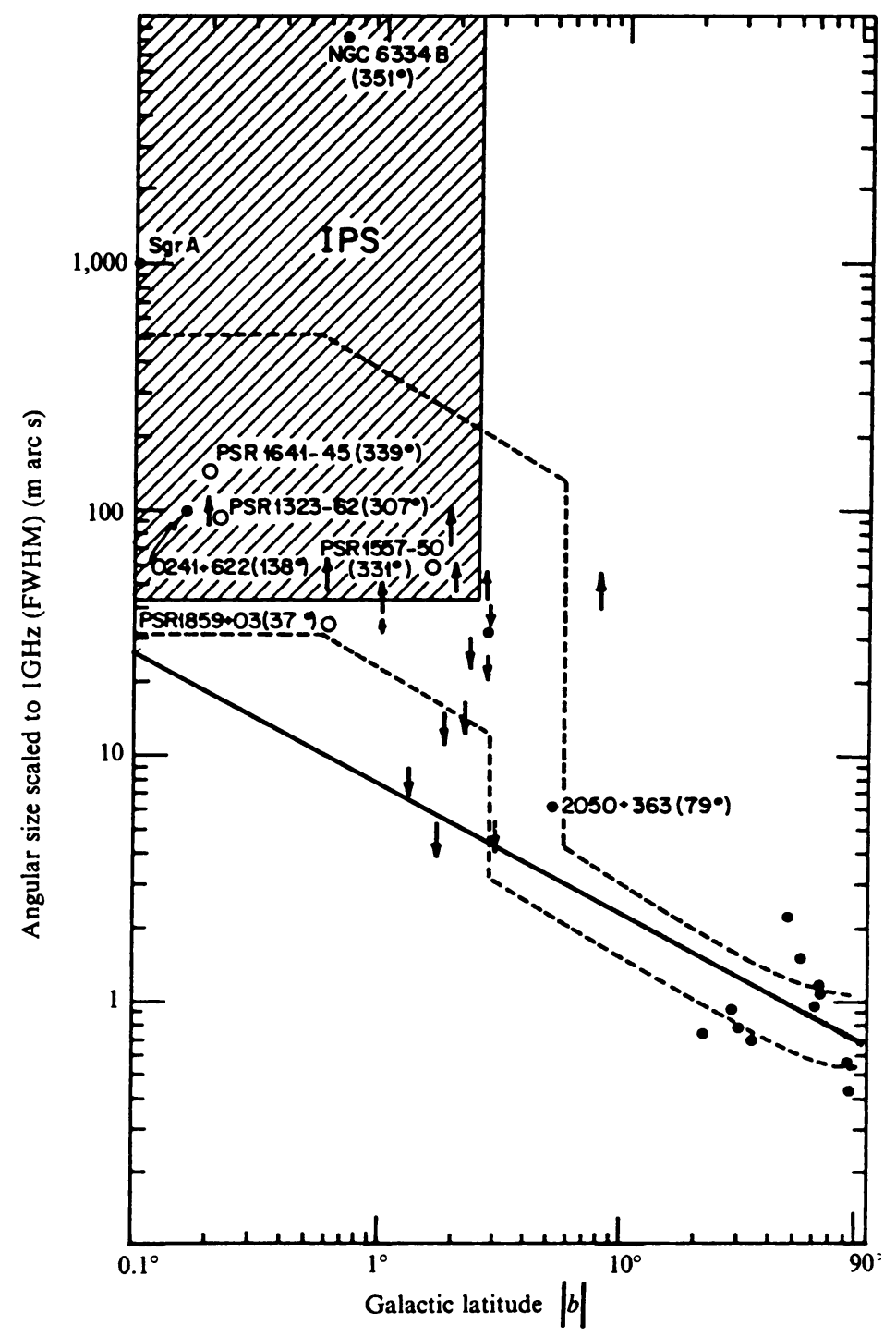

Figure 1. Scattering diameters, scaled to $1 \mathrm{GHz}$ versus magnitude of galactic latitude for the inner galaxy, from Cordes et al. (1984). The filled circles and arrows represent values and limits from VLBI at 408 MHz by Dennison et al. (1984). The point for NGC $6334 \mathrm{~B}$ is from Rodriguez et al. (1982). The open circles are implied angles for a plane wave source seen through $5-10 \mathrm{kpc}$ of scattering material, derived from particular pulsar ISS measurements and the dashed lines are from a survey of pulsar ISS (CWB). The shaded region is from interplanetary scintillation observations of sources toward the galactic center by Rao and Ananthakrishnan (1984). Note the large excursions upwards from the solid line that represents equation (1). 


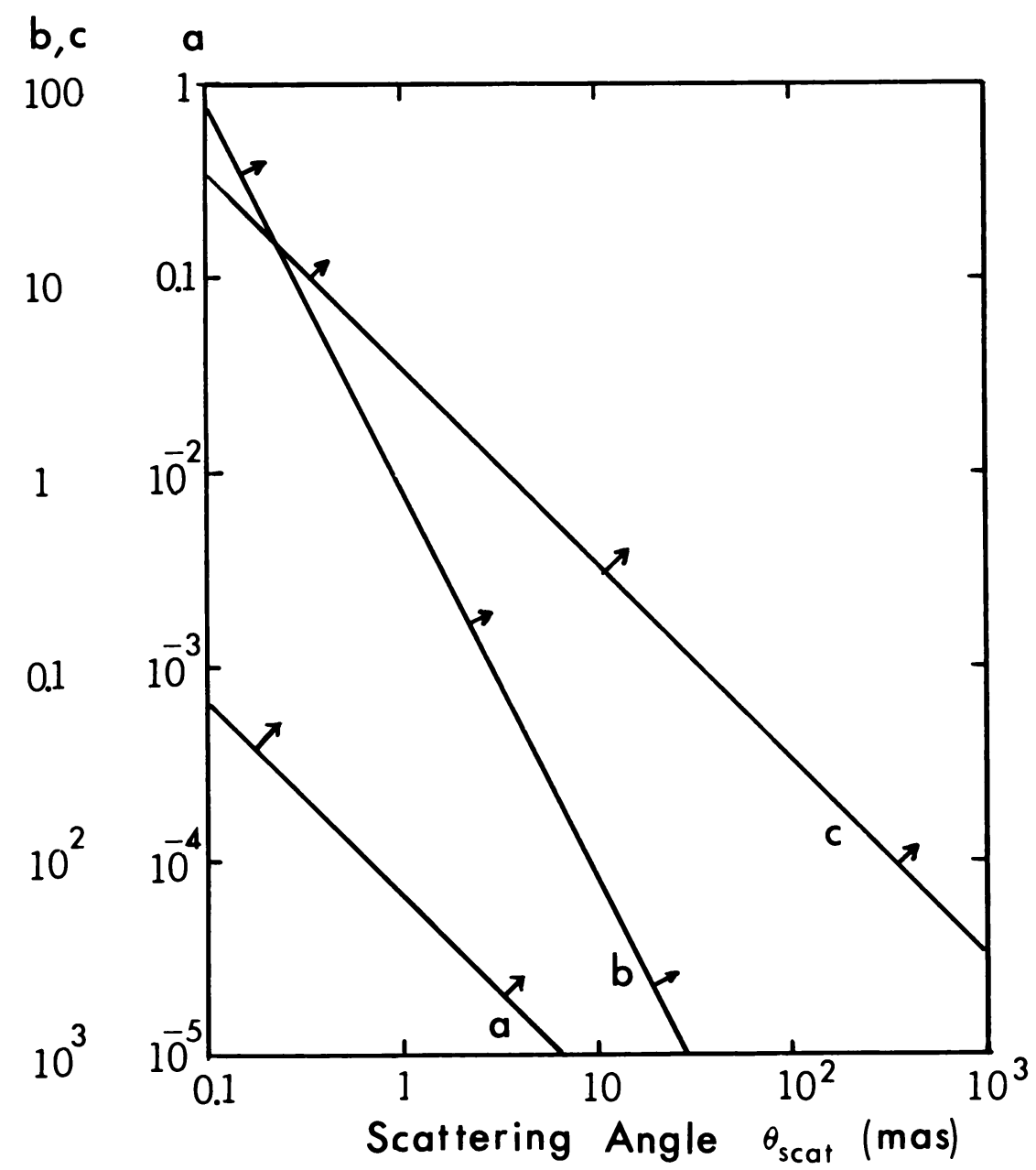

Figure 2. The various contributions to loss of visibility due to averaging of the ISS diffraction pattern, following the method of Cohen and Cronyn (1974). The baseline $\gamma$ is assumed larger than the field coherence scale $s_{0}$. The observing conditions are plotted as 3 points versus the expected scattering angle. Using scale (a) the product $\theta_{\text {source }} f_{\mathrm{GHz}} \mathrm{L}_{\mathrm{kpc}}$ (diameter in mas) is plotted; loss of visibilty occurs if the point lies above and to the right of line (a). Using scale (b) the product $\mathrm{B}_{\mathrm{MHz}} \mathrm{L}_{\mathrm{kpc}}$ is plotted; loss of visibility occurs if the point lies above and to the right of line (b). Using scale (c) the product $\mathrm{T}_{\mathrm{min}} \mathrm{f}_{\mathrm{GHz}}$ is plotted; loss of visibility occurs if the point lies above and to the right of line (c). Note that for most extra-galactic sources, source diameter averaging dominates and ensures that the visibility will be reduced by $\exp \left[-\gamma / s_{0} \beta-2\right]$. 